Recepción: 16/ 06/ 2017

Aceptación: 18 / 07/ 2017

Publicación: 15/ 11/2017

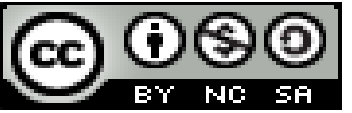

Ciencias de la educación

\title{
La psicopedagogía, una necesidad docente en la educación superior actual
}

\author{
Psychopedagogy, a teaching need in today's higher education
}

Psicopedagogia, uma necessidade de ensino na educação superior de hoje

\author{
Yelena D. Solórzano-Mendoza ${ }^{\mathrm{I}}$ \\ yelsolmen@hotmail.com
}

\section{Correspondencia: yelsolmen@hotmail.com}

I Magister en Educación y Desarrollo Social, Especialista en Diseño Curricular por Competencias, Profesora de Segunda Enseñanza Especialidad Educación Primaria, Licenciada en Ciencias de la Educación Especialidad Educación Primaria, Doctora en Ciencias de la Educación Especialización Pedagogía, Docente de la Universidad Laica Eloy Alfaro de Manabí, Manta, Ecuador. 


\title{
Resumen
}

En diferentes escenarios y espacios se habla de que la universidad debe responder a la sociedad que se vive, que se centra en la extensiva utilización de las tecnologías de información y comunicación, en las competencias laborales, en la gestión de la calidad, entre otros aspectos. En esta transformación necesaria de la universidad de hoy, los profesores juegan un papel imprescindible. Para ello, este necesita dominar los diferentes aspectos psicopedagógicos. Tomando en cuenta todo ello, el objetivo de este trabajo es revisar, mediante el análisis documental, los dominios psicopedagógicos que los docentes universitarios deben poseer con vistas a favorecer el proceso de enseñanza-aprendizaje. Se concluye que no bastan los conocimientos intelectuales y saberes específicos de los profesores que es preciso saber motivar, manejar conflictos, trabajar con la diversidad.

Palabras clave: educación superior; docentes; profesores; psicopedagogía.

\begin{abstract}
In different scenarios and spaces it is said that the university must respond to the society that lives, which focuses on the extensive use of information and communication technologies, labor competencies, quality management, among other aspects . In this necessary transformation of today's university, teachers play an indispensable role. For this, it needs to master the different psychopedagogical aspects. Taking all this into account, the aim of this work is to review, through documentary analysis, the psychopedagogical domains that university teachers must possess in order to favor the teaching-learning process. It is concluded that the intellectual knowledge and specific knowledge of the teachers is not enough that it is necessary to know how to motivate, handle conflicts, work with diversity.
\end{abstract}

Keywords: higher education; teachers; teachers; psychopedagogy.

\section{Resumo}

Em diferentes cenários e espaços, é dito que a universidade deve responder à sociedade que vive, que se concentra no uso extensivo de tecnologias de informação e comunicação, competências trabalhistas, gerenciamento de qualidade, entre outros aspectos . Nesta transformação necessária da universidade de hoje, os professores desempenham um papel indispensável. Para isso, precisa 
dominar os diferentes aspectos psicopedagógicos. Tendo tudo em consideração, o objetivo deste trabalho é rever, através da análise documental, os domínios psicopedagógicos que os professores universitários devem possuir para favorecer o processo ensino-aprendizagem. Conclui-se que o conhecimento intelectual específico e o conhecimento dos professores não são suficientes, é necessário saber como motivar, gerenciar conflitos, trabalhar com a diversidade.

Palavras chave: educação superior; professores; professores psicopedagogia.

\section{Introducción}

Según Sánchez Núñez (2001), la Universidad se contempla como una institución cuyas acciones van dirigidas básicamente al enriquecimiento intelectual, material y moral de la sociedad, a través de la formación de sus ciudadanos para el ejercicio de actividades profesionales y para la creación artística; junto con la realización de tareas de investigación y la aplicación de sus resultados.

En la "Conferencia Mundial sobre La Educación Superior en el Siglo XXI" (UNESCO, 1998), se fijaron diez principios que constituyen los fundamentos de una visión universal de la educación superior:

1. La universalidad de la educación superior supone el acceso universal para todos los que tienen las capacidades, la motivación (acceso y mérito) y la preparación adecuada en cualquier etapa de la vida.

2. La universalidad de la educación superior supone la utilización de formas variadas de intervención para atender las necesidades de educación para todos y a lo largo de toda la vida.

3. La universalidad de la educación superior supone una vocación no sólo de enseñar sino también de educar.

4. La universalidad de la educación superior supone tener una misión de vigilancia y estímulo.

5. La universalidad de la educación superior supone tener una función ética de orientación en período de crisis de valores. 
6. La universalidad de la educación superior supone que desarrolle a través de todas sus actividades una cultura de paz.

7. La universalidad de la educación superior supone desarrollar redes de solidaridad universal con otras instituciones de educación superior y con otras instituciones de la sociedad.

8. La universalidad de la educación superior supone desarrollar un modo de gestión basado en el doble principio de una autonomía responsable y una transparencia a la hora de rendir cuentas.

9. La universalidad de la educación superior supone la voluntad de explicitar los niveles de calidad y de pertinencia, más allá de los niveles concretos en determinados contextos.

10. La universalidad de la educación superior supone tener como principio axiológico, último y aglutinador de todos los demás, trabajar por la unidad de hombres y mujeres en la diferencia y complementariedad solidarias.

En este sentido, en diferentes escenarios y espacios se habla de que la universidad debe responder a la sociedad que se vive, que se centra en la extensiva utilización de las tecnologías de información y comunicación, en las competencias laborales, en la gestión de la calidad, entre otros aspectos.

En la declaración de la Conferencia Regional de Educación Superior de América Latina y el Caribe (CRES, 2008), se señala que los desafíos y retos que debemos enfrentar son de tal magnitud que, de no ser atendidos con oportunidad y eficacia, ahondarán las diferencias, desigualdades y contradicciones que hoy impiden el crecimiento de América Latina y el Caribe con equidad, justicia, sustentabilidad y democracia para la mayoría de los países que la conforman. Se valora que si bien se ha avanzado hacia una sociedad que busca cambios y referentes democráticos y sustentables, aún faltan transformaciones profundas en los ejes que dinamizarán el desarrollo de la región, entre los cuales, uno de los más importantes, es la educación y en particular la educación superior.

Ahora bien, en la transformación necesaria de la universidad de hoy, los profesores juegan un papel imprescindible. "El progreso de la Universidad está estrechamente relacionado con el 
protagonismo que genere y desempeñe cada docente en el ejercicio de su actividad profesional. Este progreso está muy vinculado a la tarea interactiva que el profesor mantiene con los estudiantes, en su objetivo de proporcionar a estos una formación adecuada a las necesidades actuales. Es, por tanto, el profesorado uno de los factores en torno al cual ha de plantearse el proceso de mejora de la calidad de la acción formativa universitaria”. (Sánchez Núñez, 2001)

Las instituciones de educación superior requieren ofrecer una educación de calidad, que es la que consigue alcanzar las metas de enseñanza, que se distinguen por su ambición y complejidad como buscar que los alumnos logren un pensamiento crítico, sean creativos y desarrollen habilidades cognoscitivas complejas. Para ello se reconoce el papel central que tiene el docente para conseguirlo. (Guzmán, 2011)

En muchos docentes es común la afirmación, de que para ser un buen profesor, basta con tener un profundo conocimiento de la ciencia o materia que imparte. Este criterio no es sólo privativo de aquellos que han llegado a su profesión sin tener una formación pedagógica previa, se escucha también entre los que sí han tenido esta preparación en sus estudios de pregrado. Como prueba palpable de lo prescindible que resulta la formación pedagógica, resultan los sobrados ejemplos de magníficos profesores que no han tenido una preparación teórica en Pedagogía, Psicología o Didáctica; sobre todo en la educación superior. Algunos llegan inclusive, a lanzar una cruzada contra las ciencias pedagógicas y se niegan rotundamente a superarse en ese campo por considerarlo algo innecesario. (Herrera Fuentes, s/f)

Con relación a esta idea resulta necesario reafirmar lo que plantean los diferentes autores (Aguilera Pupo \& Ortiz Torres, 2011) que, aunque sea convincente la necesidad de poseer docentes preparados para enfrentar esta problemática, se reconoce que generalmente no han sido formados para que organicen y dirijan este proceso.

"Es preocupante que a pesar de la situación que atraviesa la universidad haya todavía sectores educativos reacios a cualquier innovación, más preocupados en defender sus intereses corporativos que en proponer cualquier medida de mejora. Si precisamente de algo ha adolecido el sistema educativo es de la falta de formación psicopedagógica de nuestro profesorado (...) Las altas cifras de abandono y fracaso escolar en todas las etapas educativas ponen de manifiesto la 
necesidad de un cambio en la metodología docente y en la formación del profesorado (...) El profesorado necesita formación adaptada a las nuevas necesidades. Todavía no se está realizando una formación psicopedagógica de calidad que dé respuesta a los problemas que se encuentran nuestros docentes en las aulas”. (Planas Domingo, 2008)

Según (Guzmán, 2011) el profesor necesita dominar las diferentes teorías psicopedagógicas que explican el aprendizaje y la motivación.

"Actualmente, un profesional egresado, ejerce el rol de docente sin existir en su currículo de formación, ejes o dimensiones para educarlos a futuro en el perfil. Se cometen errores institucionales que agreden a la educación, cuando se contratan docentes que no tienen la suficiente preparación en docencia, en investigación, y que por ende, practican y ejercen desde otra profesión la docencia como una simple tarea de dar clases e impartir contenidos catedráticos. De allí, radica la importancia de crear una cultura interna, para educarlos, actualizarlos, motivarlos a desarrollarse como profesionales desde una disciplina para la docencia(...) Por tanto, es imprescindible que el docente universitario reciba la preparación psicopedagógica necesaria para diseñar, ejecutar y dirigir un proceso de enseñanza-aprendizaje que propicie la educación de valores. Concibiendo el proceso de enseñanza-aprendizaje como un proceso dialógico, participativo en el que docentes y estudiantes asumen la condición de personas en una dinámica transformadora de enseñanza y aprendizaje”. (Rincón Soto, 2010)

En efecto, como plantea Palomero (2003), la calidad del sistema universitario depende principalmente de la preparación de sus profesoras y profesores, quienes además de expertos en su materia deben estar en posesión de una formación psicopedagógica acorde con sus funciones y con el rendimiento que de ellos espera la sociedad. (Palomero, 2003)

Tomando en cuenta todo ello, el objetivo de este trabajo es revisar los dominios psicopedagógicos que los docentes universitarios deben poseer con vistas a favorecer el proceso de enseñanza-aprendizaje. 


\section{Metodología}

Para cumplimentar el objetivo de este trabajo se realiza el análisis documental. Primeramente se determinan las palabras claves, las estrategias de búsqueda y los recursos de información a utilizar. Se ejecutan las búsquedas en revistas relevantes relacionadas con la pedagogía y en la base de datos TESEO para localizar las tesis que responden al tema objeto de estudio. Se hizo la lectura de cada documento recuperado y se desecharon aquellos que no respondían a los objetivos de la investigación. Los pertinentes fueron analizados y sintetizados, lo que permitió inducir, deducir, comparar y organizar los contenidos.

\section{Desarrollo}

La psicopedagogía refleja interdisciplina desde su propia denominación. Es como su palabra lo indica, la confluencia de dos disciplinas: la psicología y la pedagogía. La primera dedicada a comprender al sujeto y sus procesos mentales y la segunda tiene como objeto de estudio la educación. Entonces, esto puede resumirse en: la educación abordada desde la psicología. Y el objeto de estudio es todo sujeto en situación de aprendizaje. (Contino, 2016)

Esta disciplina posee diversos campos de acción sobre los cuales puede desenvolverse un profesional psicopedagogo: institucional, educativo, de salud, laboral y clínico. (Contino, 2016)

El profesional de Psicopedagogía posee conocimientos y tiene habilidades específicas para comprender los procesos de aprendizaje y orientar a los actores implicados en el mismo; sin embargo, relativamente pocas universidades e institutos universitarios cuentan con psicopedagogos en su planta académica implicados en la formación de futuros profesionales. (Planas Domingo, 2008)

Es un hecho que los profesionales de la psicopedagogía queden vinculados desde sus orígenes a los procesos de enseñanza-aprendizaje. En este sentido, desde el Ministerio de Educación se considera al psicopedagogo como el profesional que ha de proporcionar apoyo a la tarea educativa que se desarrolla en los centros y que está relacionada con campos tales comola 
atención a la diversidad, la orientación psicopedagógica, el asesoramiento al profesorado y a otros agentes educativos. (Aznar, Cáceres, \& Hinojo, 2011)

Existe cierto consenso en identificar como sinónimos los términos orientación, orientación educativa y orientación psicopedagógica. El término orientación psicopedagógica podría englobar a los demás (orientación académica, profesional, personal, vocacional...). Se observa una progresiva tendencia a la aceptación y utilización del término orientación psicopedagógica. Los términos equivalentes orientación educativa, orientación psicopedagógica, o simplemente orientación no se identifican, pero sí comparten algunas de las características que definen la educación. (Garrido Landívar, y otros, s/f)

Existe coincidencia en la mayoría de los autores consultados acerca de que la orientación es una relación de ayuda “(...) Se considera que la relación de ayuda que se produce en la orientación no implica por parte del orientador resolver los problemas del orientando, ni eliminar por él los posibles obstáculos; sino prepararle para que por sí mismo sea capaz de superarse, adaptarse a la realidad, afrontar sus problemas, para alcanzar las metas personales y profesionales que la universidad le ha puesto y que él debe asumir como estudiante, para llegar a ser un profesional y aportar a la sociedad a la que pertenece (...) De ahí que se considere que el rol de orientador psicopedagógico solo debe ser asumido por educadores profesionales que hayan recibido la debida capacitación (...) Se trata de procesos que coinciden en el propósito de formar y desarrollar la personalidad, pero cada uno de ellos desde sus especificidades”. (Ordaz Hernández \& Márquez Marrero, 2014)

La orientación cuenta con modelos de intervención, que ofrecen, a su vez, distintas posibilidades de acción. Nos referiremos a "intervención” como las estrategias que se emplean para conseguir unos resultados propuestos. (Primogerio, 2008)

Algunos ejemplos de la intervención psicopedagógica se muestran en la literatura:

- Cómo trabajar con el alumno difícil en el aula.

- $\quad$ Proporcionar consejos en forma particular y/o grupal, formal y/o informal.

- $\quad$ Espacios de orientación para alumnos con dificultades académicas. 
- Análisis de deserción y desgranamiento de alumnos.

- Atención a alumnos del interior y del exterior.

- $\quad$ Entre otras.

Los profesores deben, según algunos autores (Guzmán, 2011):

- Estar consciente acerca de las diversas características físicas, sociales y psicológicas de sus alumnos.

- Conocer quién es el aprendiz y cómo ocurre el proceso de aprendizaje.

- $\quad$ Promover en sus estudiantes la comprensión más que la recepción pasiva de saberes.

- Ayudar a los estudiantes a autorregular su aprendizaje.

- Motivar a sus estudiantes explicitando los beneficios que obtendrán si adquieren lo enseñado.

- Corregir las realizaciones de los estudiantes.

- Enseñar a trabajar cooperativamente a sus estudiantes.

- Enseñar a sus estudiantes a ser críticos.

- Enseñar a sus estudiantes a empatizar.

- Identificar las diferentes clases de ideas previas y preconcepciones que por lo regular tienen los estudiantes, y entonces encaminar su enseñanza a transformarlas.

Es preciso que los docentes a nivel psicopedagógico dominen, a partir de la literatura consultada (Garrido Landívar, y otros, s/f):

- Dar clases a grupos heterogéneos, con diferentes capacidades y rendimiento.

- Trabajar con los alumnos disruptivos, la desmotivación, el déficit de atención, la hiperactividad, las ludopatías, la anorexia, la bulimia, el ciber bullying o la drogadicción.

- Manejar la diversidad, los agrupamientos más flexibles, las nuevas tecnologías aplicadas a la educación.

- Mejorar la convivencia.

- Dinamizar un aula.

- Llevar a cabo una entrevista. 
- Resolver conflictos en el aula.

Palomero (2003), considera que los profesores universitarios deben cambiar la mentalidad, esforzarse en atraer el interés del alumno, disponerse a renovarse, a investigar nuevas formas del quehacer psicopedagógico y a demostrar en las aulas, en el seminario, en el laboratorio o en cualquier otro escenario su capacidad para comunicar, para motivar, para transmitir, para enseñar. "Sean matemáticos, físicos, historiadores, médicos, químicos, ingenieros, psicólogos, abogados, o incluso pedagogos o literatos, con frecuencia tienen dificultades para despertar el interés de sus alumnas y alumnos y para transmitir con eficacia su patrimonio científico e intelectual. Disfrutan investigando y construyendo el conocimiento de la realidad, pero encriptan el lenguaje, hablan y escriben para iniciados, tienen dificultades para hacerse entender; en una palabra, despilfarran sus conocimientos, que más que suyos son patrimonio de la humanidad. Quizá a muchos de ellos/as no les enseñó nadie a presentar atractivamente sus saberes, ni a exponer con claridad, ni a conectar, convencer y comunicar. (...) He aquí uno de los grandes retos a resolver por la Universidad en el momento actual”. (Palomero, 2003)

\section{Conclusiones}

Es evidente que el profesorado debe tomar en cuenta la psicopedagogía para su labor. Gran parte del malestar docente se debe a la falta de esta competencia, que debe ser previa al resto de las competencias que debe tener el profesor. Si un profesor no posee recursos para dinamizar un grupo, llevar a cabo una entrevista o resolver un conflicto de convivencia, todo su conocimiento intelectual y científico no le va hacer muy factible delante de un aula.

Es preciso la formación psicopedagógica del profesor universitario como orientador para contribuir a elevar la calidad de la formación integral de la personalidad de los futuros egresados y enfrentar los retos de la educación superior en la compleja realidad existente. 


\section{Referencias bibliográficas}

Aguilera Pupo, E., \& Ortiz Torres, E. (2011). El nivel psicopedagógico en docentes universitarios, su repercusión en los perfiles de estilos de aprendizaje. Revista Estilos de Aprendizaje 7 (7).

Contino, M. (2016). La psicopedagogía a modo de prevención. Importancia en la detección temprana de dificultades para lograr el proceso de lectoescritura. Trabajo Final para optar por la Licenciatura en Psicopedagogía.

CRES. (2008). Declaración de la Conferencia Regional de Educación Superior en América Latina y el Caribe. Citado por: Aguilera Pupo, E.; Ortiz Torres, E. El nivel psicopedagógico en docentes universitarios, su repercusión en los perfiles de estilos de aprendizaje. Revista Estilos de Aprendizaje, $\mathrm{n}^{\circ} 7$, Vol. 7, abril de 2011.

Garrido Landívar, J., Suárez Ramírez, J., Cabello Naranjo, M., Díaz Bolaños, C., Oliva Metola, M., Almeida Rivero, T., \& González Peñate, F. (s/f). Orientaciones psicológicas, psicopedagógicas y sociales para los estudiantes durante la vida académica en la universidad de las palmas de gran canaria.

Guzmán, J. (2011). La calidad de la enseñanza en educación superior ¿Qué es una buena enseñanza en este nivel educativo? Perfiles educativos, 33.

Herrera Fuentes, J. (s/f). Psicopedagogía en el profesorado. Recuperado el 06 de febrero de 2016, de http://www.psicopedagogia.com/

Primogerio, C. (2008). Intervención psicopedagógica en la universidad. Congreso Internacional de Psicopedagogía.

Sánchez Núñez, J. (2001). Necesidades de formación psicopedagógica para la docencia universitaria. Memoria para optar al grado de doctor. 
UNESCO. (1998). La educación superior en el siglo XXI: visión y acción. Conferencia Mundial sobre la Educación Superior. París: Conferencia Mundial sobre la Educación Superior. 